\title{
Characterisation of Retinal Feature Points Applied to a Biometric System
}

\author{
David Calvo, Marcos Ortega, Manuel G. Penedo, José Rouco, \\ and Beatriz Remeseiro
}

\begin{abstract}
VARPA Group, Department of Computer Science, University of A Coruña, Spain \{dcalvo, mortega, mgpenedo, jrouco, bremeseiro\}@udc.es
\end{abstract}

\begin{abstract}
In this work a methodology for the classification of retinal feature points is applied to a biometric system. This system is based in the extraction of feature points, namely bifurcations and crossovers as biometric pattern. In order to compare a pattern to other from a known individual a matching process takes place between both points sets. That matching task is performed by finding the best geometric transform between sets, i.e. the transform leading to the highest number of matched points. The goal is to reduce the number of explored transforms by introducing the previous characterisation of feature points. This is achieved with a constraint avoiding two differently classified points to match. The empirical reduction of transforms is about $20 \%$.
\end{abstract}

Keywords: Retinal verification, Feature points characterisation, Registration.

\section{Introduction}

Retinal vessel tree pattern has been proved a valid biometric trait for personal authentication as it is unique, time invariant and very hard to forge, as showed by Mariño et al. [1], who introduced a novel authentication system based on this trait. In that work, the whole arterial-venous tree structure was used as the feature pattern for individuals. One drawback of the proposed system was the necessity of storing and handling the whole vessel tree image as a pattern. Based on the idea of fingerprint minutiae 2, a more ideal and robust pattern was introduced and analysed in [34] where a set of landmarks (bifurcations and crossovers of retinal vessel tree) were extracted and used as feature points. In this scenario, the pattern matching problem is reduced to a point pattern matching problem. The matching is achieved by exploring the transform space to find the optimal transform in which the most points from both patterns are matched. The search is performed by forcing two matches between patterns, calculating the transform associated and calculating the additional matched produced. The matching between two points is accepted if once the transform is applied, both points are close enough.

This approach implies the exploration of a high number of transformations. The method proposed in this paper, classifies the feature points in the two 
possible classes (bifurcations or crossovers) and introduces a constraint where two points with different characterisation can not match. This will allow to reduce the exploration in the transform space.

In the bibliography there are some works that approached this problem of characterisation of retinal points. The methods were not applied to a specific domain and the image processing techniques were more heavy computationally. For instance, the method proposed by Chia-Ling Tsai et al. [5], use vessel segments intersections as seeds to track vessel centre lines and classify feature points according to intersection angles. Another work, the proposed by V.Bevilacqua et al. [6], uses a small window to analyse the whole skeleton of the vascular structure. The main problem of this solution is the misclassification of crossovers, as they are only properly classified when the vessel segments intersect exactly in the same pixel. In order to correctly characterise the feature points, the methodology in this work takes advantage of the pattern extraction process and does not require additional processing of the original image. Feature points are classified according to a local analysis and a topological study.

The paper is organised as follows: in section 2 a general view of the retinal biometric system is explained. Section 3 introduces the methodology for characterisation of feature points. Section 4 shows the results of several experiments to test the classification performance and its impact in the biometric system. Finally, section 5 discusses some conclusions.

\section{Retinal Recognition System}

In the first step, to obtain a good representation of the tree, the creases of the image are extracted [7]. As vessels can be thought of as ridges or valleys seeing the retinal image as a landscape, creases image will consist in the vessels skeleton (Figure 1).

Using the whole creases image as biometric pattern has a major problem in the codification and storage of the pattern, as we need to store the whole image. To solve this, similarly to the fingerprint minutiae (ridges, endings, bifurcations
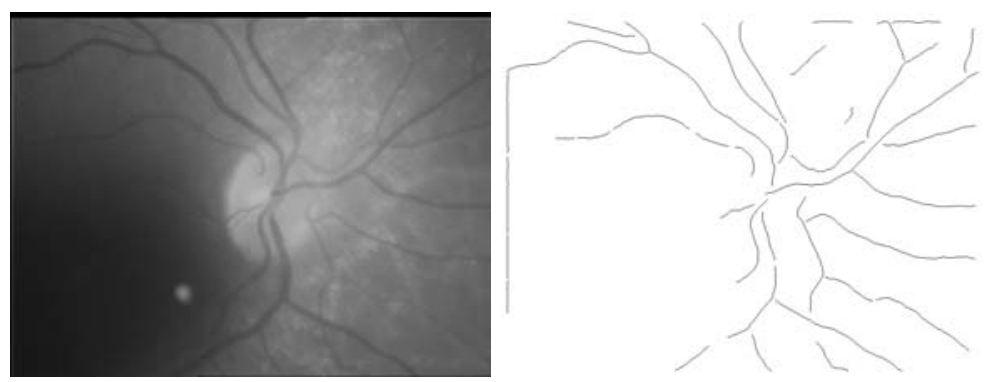

Fig. 1. Left, original image; Right, creases image extracted from original 
in the fingerprints), the biometric pattern consists in a set of landmarks from the creases image. The most prominent landmarks in retinal vessel tree are crossovers (between two different vessels) and bifurcation points (one vessel coming out of another one). These are the feature points considered in the authentication system.

\subsection{Biometric Pattern Extraction}

To detect feature points, creases are tracked to label all of them as segments in the vessel tree, marking their endpoints. Next, bifurcations and crossovers are detected by means of relationships between endpoints. These relationships are extracted by finding segments endpoints close enough to each other and analysing orientations.

Due to quality or illumination issues in the images, there could be some discontinuities along a vessel so a relationship of union between segments must be considered to avoid detection of false feature points.
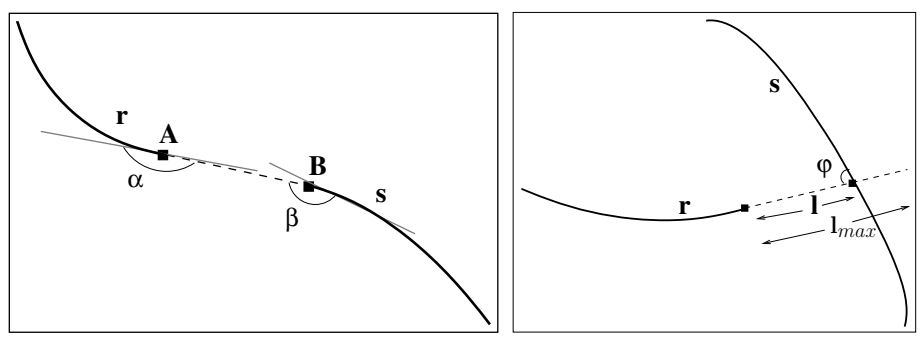

Fig. 2. Left, representation of an union relationship between segments $r$ and $s$. Endpoints $A$ and $B$ are connected through a straight line and both their angles with that line are computed $(\alpha$ and $\beta$ ). Right, representation of a bifurcation between segments $r$ and $s$. Segment $r$ is prolongated for a maximum length $l_{\max }$ and, eventually, $s$ is found forming a $\phi$ angle with $r$ original endpoint direction.

An union happens when two adjacent segments belong to the same vessel. To detect an union between two segments, $r$ and $s$, these are connected through a straight line between their closest endpoints (Figure 2, left). The angles formed by both endpoint directions and the straight line are calculated. Both angles should be closed to $\pi$ radians to accept it as a union. A threshold $\theta$ is defined. To obtain the bifurcations, segments are prolongated through their endpoints direction to a maximum fixed length $\left(l_{\max }\right)$. If a pixel of another segment is found, the bifurcation is marked and the angle formed by both segments is calculated (Figure 2, right). Note that this method also allows to detect crossovers as they can be seen as bifurcations by both sides. Figure 3 shows two examples of biometric patterns obtained. 


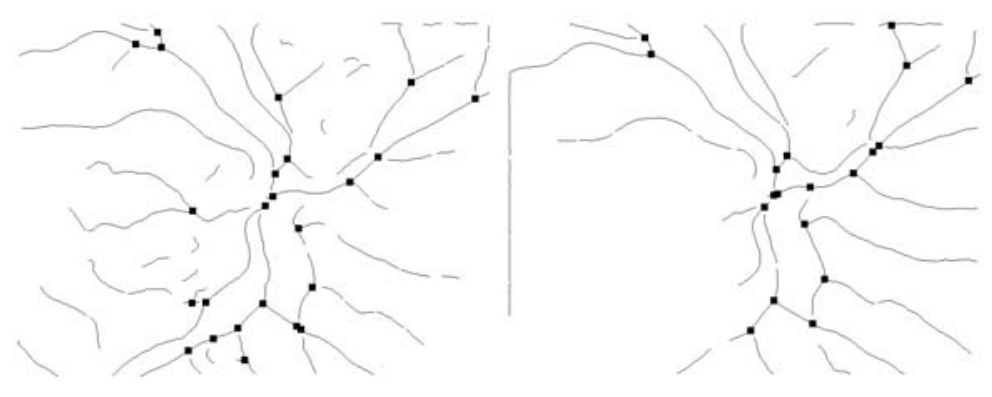

Fig. 3. Examples of feature points obtained in creases from the same individual extracted from images acquired in different times

\subsection{Biometric Patterns Matching}

In the matching process, the biometric pattern $\nu$ stored for a particular individual is compared to the pattern $\nu^{\prime}$ extracted in the previous section when a user claiming that identity tries to be verified. Due to the eye movement during the image acquisition stage, it is necessary to align $\nu$ with $\nu^{\prime}$ in order to be matched 8. This movement of the eye consists basically only in translation in both axis, rotation and sometimes a very small change in scale. Considering this, a Similarity Transform schema (ST) is used to model pattern transformations [9] It is also important to note that both patterns, $\nu$ and $\nu^{\prime}$ could have a different number of points as showed in Figure 3 .

To find the best transformation (i.e. the one with the highest similarity value associated, for a given similarity metric) a search is performed in the transformation space. In ST model, two points of each pattern are necessary to compute the transformation parameters and, thus, the maximum transformation space size $(T)$ is given:

$$
T=\frac{\left(M^{2}-M\right)\left(N^{2}-N\right)}{2}
$$

where $M$ and $N$ represent the cardinality of $\nu$ and $\nu^{\prime}$ respectively. Once both patterns are aligned, a point $p$ from $\nu$ and a point $p^{\prime}$ from $\nu^{\prime}$ match if distance $\left(p, p^{\prime}\right)<$ $D_{\max }$, where $D_{\max }$ is a threshold introduced in order to consider the quality loss and discontinuities during the creases extraction process leading to mislocation of feature points by some pixels. Therefore, for a given transformation the number of matched points between patterns can be calculated. Using this information, a similarity metric must be established to obtain a final criterion of comparison between patterns. In [10] a similarity measure between patterns, $S_{\gamma}$, is introduced based on a $\gamma$ parameter:

$$
S_{\gamma}=\frac{C^{\gamma}}{\sqrt{M N}}
$$


where $C$ is the number of matched points between patterns, $M$ and $N$ is the size of both patterns and $\gamma$ is a parameter controlling the influence of matched points information.

The matching process weakness is the high amount of transformations that need to be calculated to find the optimal one. To reduce the number of calculations, more information about the domain is introduced. By characterising the feature points into crossovers and bifurcations, an efficient constraint can be added only allowing points of the same class (or unclassified) to match.

\section{Feature Points Characterisation}

This classification is done according to local features of those points and a topological analysis between them.

The first classification step is done according to local features of the points without considering, for it, the effect of the other points. So, to define a classification for a point the number of vessel segments that create the intersection is studied. Each detected feature point, $F$, is used as centre of a circumference with radius $R_{c}$ used for the analysis. $n(F)$ gives the number of vessel segments that intersect the circumference being the point, $F$, such as a bifurcation corresponds to $n(F)=3$ and a crossover to $n(F)=4$. Fig 4 shows these two possible classifications. The images shows the blood vessels, the circumference used to do the analysis, and, coloured darker, the pixels where the vessels intersect the circumference.

By increasing $R_{C}$ too much the circumference can be intersected by vessels that do not belong to the feature point. To avoid this problem a vote system with three radius sizes is used: $R_{c}$ and two new radius defined around $R_{c}, R_{1}=R_{c}-\rho$ and $R_{2}=R_{c}+\rho$. In this work, $\rho=5$. With these definitions, two values are calculated, $C(F)$ and $B(F)$, meaning the number of votes for a point $F$ to be classified, respectively, as a crossover and a bifurcation:

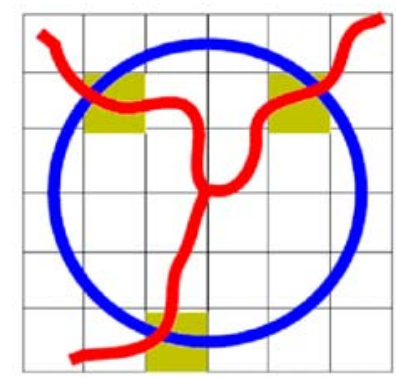

(a)

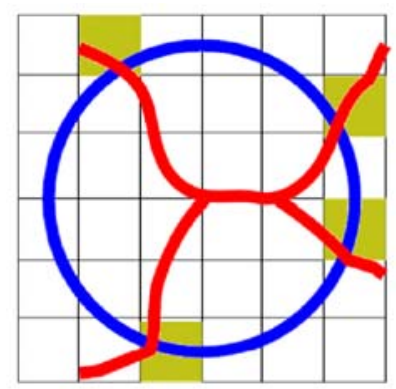

(b)

Fig. 4. Preliminary feature point classification according to the number of vessel intersections where (a) represents a bifurcation and (b) a crossover 


$$
\begin{aligned}
& C(F)=2 * C\left(F, R_{1}\right)+C\left(F, R_{c}\right)+C\left(F, R_{2}\right) \\
& B(F)=B\left(F, R_{1}\right)+B\left(F, R_{c}\right)+2 * B\left(F, R_{2}\right)
\end{aligned}
$$

where $C\left(F, R_{i}\right)$ and $B\left(F, R_{i}\right)$ are binary values indicating if $F$ is classified, respectively, as a crossover or a bifurcation using a radius $R_{i}$. Note that the contribution of the small radius is more valuable, and therefore weighted, in the crossover classification while for bifurcations the big radius adds more information. $F$ will be classified as a crossover when $C(F)>B(F)$ and a bifurcation otherwise.

Due to the representation of crossovers in the skeleton, this information is not enough to assure that a feature point is a crossover while being a necessary condition. According to this, a topological classification is needed analysing the feature points in pairs, $\left(F_{1}, F_{2}\right)$, attending to their Euclidean distance $d\left(F_{1}, F_{2}\right)$. If both $F_{1}$ and $F_{2}$ are connected by a vessel segment and $d\left(F_{1}, F_{2}\right)<=2 * R_{c}$, both points are merged into a crossover in the middle point between them.

For the rest of feature points not classified as crossovers, it can not be guaranteed they are bifurcations as for every real crossover not classified, two false bifurcations would be created. Because of this, it is necessary to use another threshold $\left(R_{b}\right)$ to allow to take a decision of which points are accepted as bifurcations. This process is analogous to the previously presented for crossover classification. In this way, for each bifurcation its pair, understood as the connected bifurcation that minimises the Euclidean distance, is found. For each pair, a circumference with radius $\left(R_{b}\right)$ centred in the middle point of the segment between the points is used. This circumference cannot contain both points.

Every pair of points not fulfilling the conditions is marked as not classified in the final result. This is due to the fact that points are not close enough to be considered as one crossover but not far enough to be considered as two independent bifurcations.

Note that $R_{c}$ and $R_{b}$ parameters allow to tune the system in terms of specificity and sensitivity as some domains would require different performances. In the next section, some experiments and performance results are shown.

\section{Results}

To perform the experiments a set of 30 images from 15 different individuals from VARIA database 11 were used. These images are centred in the optic disc with spatial resolution of $768 \times 584$ pixels and were labelled by medical experts to test the performance in the classification of feature points.

In the first test, the performance of the classification system was evaluated. The performance analysis is made as a function of $R_{c}$ and $R_{b}$ as they are the parameters that allow to tune the specificity and sensitivity of the system. specifically, $R_{c}$ makes a direct impact in the crossover classification while $R_{b}$ makes it in the bifurcations. For a right choice of the mentioned parameters a quantitative study according to the parameters is presented. The results allow to choose 


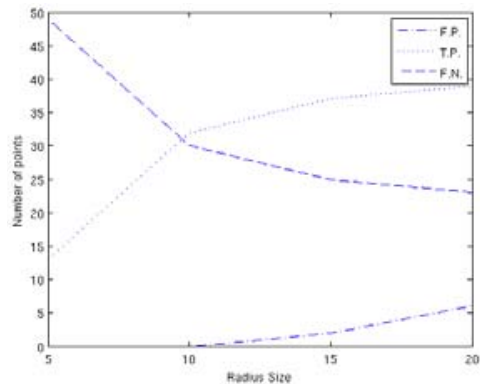

(a)

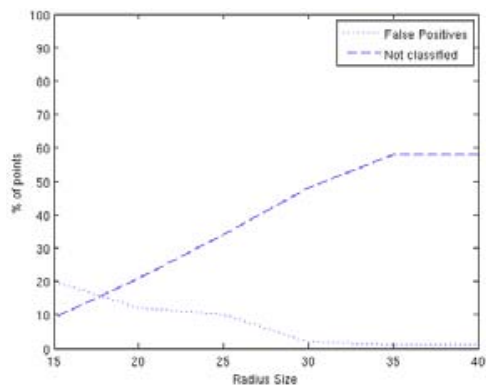

(b)

Fig. 5. Analysis of the influence of $R_{c}$ for the crossovers, (a), and $R_{b}$ for the bifurcations, (b) in the classification performance of the system

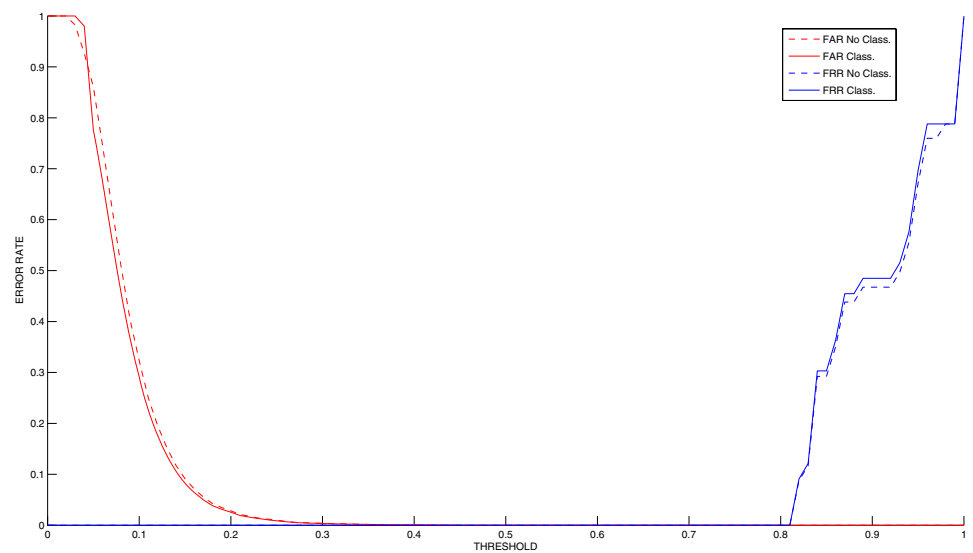

Fig. 6. FAR and FRR curves for using the test set. The system classification capability remains almost identical.

the adequate parameters for a specific domain where the desired sensitivity or specificity levels can change depending on the False Positives, True Positives and False Negatives as shown in Fig $5(\mathrm{a})$

Fig 5(b) shows the results for bifurcation classification according to the chosen $R_{b}$ radius. This figure displays a new category, the non classified points, that includes the points that fulfilling the morphology conditions are not close enough to be classified as crossover and not far enough not to be classified as independent bifurcations. The bigger radius, $R_{b}$, used the more number of points without classifying but the number of false positives will be below the $1 \%$. Opposite to this, if a big level of true positives is needed with a small radius the sensitivity is over the $70 \%$. Selecting $R_{c}=10$ and $R_{b}=30$, the global sensitivity of the system is $67 \%$ and the specificity $95 \%$. The specificity is a highly important parameter 
Table 1. Statistics on the transformations removed in the matching process. The columns refer to total possible transformations without considering the point classification restriction, the number of transformations avoided by including the restriction and the mean and standard deviation (std) percentages of transformation removed per image.

\begin{tabular}{lccc}
\hline Total & Removed & Mean & Std \\
\hline 38617 & 7238 & $18.74 \%$ & $5.97 \%$ \\
\hline
\end{tabular}

in this domain as a low rate indicates the chance to match two different points. This could lead to a bad performance of the recognition system.

In the second experiment this mentioned biometric system performance using the characterisation of points is tested. The goal is to check if the recognition rate is not diminished by the feature points classification errors. All the test images were compared versus all. Fig. 6] shows the False Acceptance (FAR) and Rejection (FRR) rate curves for the biometric system with and without using classification of feature points.

The classification performance of the system remains the same while reducing the computation load of the matching process by avoiding matches between differently classified points. This constraint removes a lot of irrelevant transformations from the computation. Table 1 shows the impact in the test set in terms of transformations removed. The average reduction of computed transformations was $18.74 \%$.

\section{Conclusions}

In this work a methodology for characterisation of retinal vessel tree feature points is applied into a matching stage for a retinal recognition system. The matching process of two biometric patterns, i.e. two sets of feature points, involves the calculation of many possible similarity transforms where points from both patterns are matched. By characterising the points into crossovers or bifurcations, a constraint is added where two differently classified points can not match.

The methodology employed for the characterisation of points allows to tune the sensitivity and specificity values. Particularly in this domain, a high specificity is very valuable as this indicates the rate of misclassified points. The systems shows a specificity value of $95 \%$. Because of this, the biometric system is improved in terms of computational optimisation while not reducing the high recognition rate.

In terms of transform operations reduction, the system computes a $18.74 \%$ less in the matching stage. The average execution time of the whole process in Pentium 4 Core Duo 2.4Ghz Desktop PC was $135 \mathrm{~ms}$, meaning a $12.78 \%$ faster than before adding the characterisation constraint. This supposes a great advantage specially in identification tasks where the same biometric pattern is compared with all the stored ones in the database of known users. 


\section{References}

1. Mariño, C., Penedo, M.G., Penas, M., Carreira, M.J., González, F.: Personal authentication using digital retinal images. Pattern Analysis and Applications 9, $21-33(2006)$

2. Tan, X., Bhanu, B.: A robust two step approach for fingerprint identification. Pattern Recognition Letters 24, 2127-2134 (2003)

3. Ortega, M., Mariño, C., Penedo, M.G., Blanco, M., González, F.: Personal authentication based on featue extraction and optica nerve location in digital retinal images. WSEAS Transactions on Computers 5(6), 1169-1176 (2006)

4. Ortega, M., Penedo, M.G., Mariño, C., Carreira, M.J.: Similarity metrics analysis for feature point based retinal authentication. In: Campilho, A., Kamel, M.S. (eds.) ICIAR 2008. LNCS, vol. 5112, pp. 1023-1032. Springer, Heidelberg (2008)

5. Tsai, C.L., Stewart, C., Tanenbaum, H., Roysam, B.: Model-based method for improving the accuracy and repeatability of estimating vascular bifurcations and crossovers from retinal fundus images. IEEE Transactions on Information Technology in Biomedicine 8(2), 122-130 (2004)

6. Bevilacqua, V., Cambó, S., Cariello, L., Mastronardi, G.: A combined method to detect retinal fundus features. In: Proceedings of IEEE European Conference on Emergent Aspects in Clinical Data Analysis (2005)

7. López, A., Lloret, D., Serrat, J., Villanueva, J.: Multilocal creasness based on the level set extrinsic curvature. Computer Vision and Image Understanding 77, 111-144 (2000)

8. Zitová, B., Flusser, J.: Image registration methods: a survey. Image Vision and Computing 21(11), 977-1000 (2003)

9. Ryan, N., Heneghan, C., de Chazal, P.: Registration of digital retinal images using landmark correspondence by expectation maximization. Image and Vision Computing 22, 883-898 (2004)

10. Ortega, M., Penedo, M.G., Mariño, C., Carreira, M.J.: A novel similarity metric for retinal images based authentication. In: International Conference on Bio-inspired Systems and Signal Processing, vol. 1, pp. 249-253 (2009)

11. VARIA: Varpa retinal images for authentication, http: //www . varpa.es/varia.html 